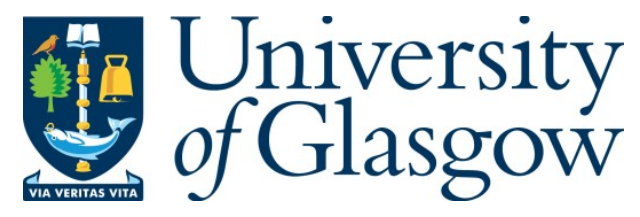

Abdrabou, Y., Mostafa, M., Khamis, M. and Elmougy, A. (2019) Calibration-free Text Entry Using Smooth Pursuit Eye Movements. In: 11th ACM Symposium on Eye Tracking Research and Applications, Denver, CO, USA, 25-28 June 2019, p. 35. ISBN 9781450367097 (doi: 10.1145/3314111.3319838).

There may be differences between this version and the published version. You are advised to consult the publisher's version if you wish to cite from it.

(C) The Authors 2019. This is the author's version of the work. It is posted here for your personal use. Not for redistribution. The definitive Version of Record was published in the Proceedings of the 11th ACM Symposium on Eye Tracking Research \& Applications (ETRA '19), Denver, Colorado, USA, 25-28 Jun 2019, Article 35. ISBN 9781450367097. https://doi.org/10.1145/3314111.3319838.

http://eprints.gla.ac.uk/181988/

Deposited on: 19 March 2019

Enlighten - Research publications by members of the University of Glasgow http://eprints.gla.ac.uk 


\title{
Calibration-free Text Entry using Smooth Pursuit Eye Movements
}

\author{
Yasmeen Abdrabou ${ }^{1,2}$, Mariam Mostafa ${ }^{1}$, Mohamed Khamis ${ }^{3}$, Amr Elmougy ${ }^{4}$ \\ ${ }^{1}$ German University in Cairo, Egypt, \{firstname.lastname\}@guc.edu.eg \\ ${ }^{2}$ Bundeswehr University, Munich, Germany, yasmeen.abdrabou@unibw.de \\ ${ }^{3}$ University of Glasgow, United Kingdom, Mohamed.Khamis@glasgow.ac.uk \\ ${ }^{4}$ University of Canada in Egypt, Egypt, \{firstname.lastname\}@uofcanada.edu.eg
}

\begin{abstract}
In this paper, we propose a calibration-free gaze-based text entry system that uses smooth pursuit eye movements. We report on our implementation, which improves over prior work on smooth pursuit text entry by 1) eliminating the need of calibration using motion correlation, 2) increasing input rate from 3.34 to 3.41 words per minute, 3) featuring text suggestions that were trained on 10,000 lexicon sentences recommended in the literature. We report on a user study $(\mathrm{N}=26)$ which shows that users are able to eye type at 3.41 words per minutes without calibration and without user training. Qualitative feedback also indicates that users positively perceive the system. Our work is of particular benefit for disabled users and for situations when voice and tactile input are not feasible (e.g., in noisy environments or when the hands are occupied).
\end{abstract}

\section{CCS CONCEPTS}

- Human-centered computing $\rightarrow$ Human computer interaction (HCI); Text input.

\section{KEYWORDS}

Calibration-Free, Text Entry, Gaze Interaction, Smooth Pursuits

\section{ACM Reference Format:}

Yasmeen Abdrabou ${ }^{1,2}$, Mariam Mostafa ${ }^{1}$, Mohamed Khamis ${ }^{3}$, Amr Elmougy ${ }^{4}$. 2019. Calibration-free Text Entry using Smooth Pursuit Eye Movements. In 2019 Symposium on Eye Tracking Research and Applications (ETRA '19), fune 25-28, 2019, Denver, CO, USA. ACM, New York, NY, USA, 5 pages. https://doi.org/10.1145/3314111.3319837

\section{INTRODUCTION AND RELATED WORK}

New technologies are explored to enhance the user's experience and to maximize the interaction of the user, especially in public displays. One of the most popular interaction technologies is eye trackers. Using eye tracking makes it easier to see through a user's eyes, to know what catches the user's attention and to know more about the user and his/her behavior. With the new advancements in eye tracking techniques, it became more affordable. However, it still suffers from the calibration problem.

Permission to make digital or hard copies of all or part of this work for personal or classroom use is granted without fee provided that copies are not made or distributed for profit or commercial advantage and that copies bear this notice and the full citation on the first page. Copyrights for components of this work owned by others than the author(s) must be honored. Abstracting with credit is permitted. To copy otherwise, or republish, to post on servers or to redistribute to lists, requires prior specific permission and/or a fee. Request permissions from permissions@acm.org.

ETRA '19, June 25-28, 2019, Denver, CO, USA

(C) 2019 Copyright held by the owner/author(s). Publication rights licensed to ACM. ACM ISBN 978-1-4503-6709-7/19/06 ..\$15.00

https://doi.org/10.1145/3314111.3319837
Calibration process is considered to be tedious, time-consuming for the user and memory space consuming for the application/system. The consumed time and the memory space during calibration is bearable (but not desirable) for an application with a small number of users or for a desktop application. But for a public displays' system which mainly depends on immediate usability by many users with short interaction times, it is not acceptable at all. A better use is to have a calibration-free system deployed on public displays $[5,6]$. It also can be used where tactile or voice interaction is not possible, hence calibration is still an issue.

Since the human eyes and gaze behavior reflects cognitive processes and can give hints of our thinking and intentions as they reveal a lot about the user's interests and behavior. It is also said that that humans often look at things before taking action [8]. This means that using human eyes in interaction will be fast and reliable as the eyes always look at what they want or what seems interesting for the user. Therefore, there is a wide range of application areas which make use of the eye-tracker. Eye-trackers is used in marketing [22], usability in human-computer interaction [15], psychology [17], gaming, etc and text entry is not an exception. Research has been done for using gaze as a text entry methodology $[3,7,12,14,18-20]$. It was also used by removing the calibration process by doing a one-point calibration technique [9]. However, no calibration free applications have been implemented yet.

Accordingly, in this paper, we introduce a calibration-free gaze based text entry using smooth pursuits. This application does not take extra time for calibration, hence reduce the wasted time. It uses motion correlation for this purpose where the user traces the moving letters by gaze. We achieved 3.41 WPM unlike the literature using the same layout with 3.34 WPM.

\section{DESIGN AND IMPLEMENTATION}

In this paper, we describe a calibration-free gaze-based text entry using smooth pursuits. The system determines which object the user is looking at by correlating eye movements and object movements. When the system detects eyes on the screen the objects start rotating, when the eyes follow the rotation path, a correlating is being calculated using the dynamic objects on the display with the smooth pursuit eye movement performed when the eyes follow a moving object. By using an eye-tracking device, a stream of the user's gaze points (XY-coordinates) will be collected, as well as the positions of the application's objects on the screen (XY-coordinates). Having these stream of XY-coordinates and by computing the correlation between each of the objects' positions with the gaze points, in the X-axis and in the Y-axis separately. If an object has a correlation with the gaze points $>0.9$ in the $\mathrm{X}$-axis and in the $\mathrm{Y}$-axis, then 
this object will be selected. Otherwise, no object will be selected and the system will collect more points and correlate again till one of the object's correlation values pass the threshold. We used math.net library to calculate the correlation [13].It is important to note that the eye tracker was calibrated at the beginning until the Tobii software reported accurate tracking ${ }^{1}$.

\subsection{User Interface}

This eye-controlled text entry system is an approach towards a calibration-free eye-based text entry which is suitable for public displays. The system combines a two-stage interface concept $[2,9]$ with interaction designed specifically for a calibration-free approach using smooth pursuit movements. The detection mechanism used is related to the approach used in the study made by Khamis et al [6]. The layout of the system will follow the layout proposed by Lutz, Venjakob, and Ruff in their text entry system using eye pursuits [9]. The system's interaction is designed to provide a calibration-free application which will make use of the smooth pursuit eye movements and its characteristics [6].

\subsection{Layout Design}

The layout used is similar to the SMOOVS text entry system [9], which is implemented similarly to the layout of the EEG3-based Hex-O-Spell, which is a mental text entry system [4]. The EEG3based Hex-O-Spell layout is a hexagonal layout with hexagonal tiles on it, the layout is used in this system will be typical to SMOOVS (as shown in figure 1a).

The user interface used in the application is listing all of the English alphabets, backspace, space and some special characters which are full-stop, comma, exclamation mark, and question mark. The reason behind the division of the layout into six objects is that this division approach which is supported by a smooth pursuitbased interaction [21] where the detection rate from four to six objects was high and similar to each other. But when the number of presented objects exceeds six, the detection rate drops. This system is using six interactive clusters, a cluster is a group of six hexagon where each hexagon holds a character. Each cluster holds up six neighboring letters from the alphabets, and the last cluster of hexagons will contain the $\mathrm{Y}, \mathrm{Z}$ and some special characters which are (., ! ?). At the center of the screen, an empty white text box is placed. Which holds the selected characters by the user, and it is placed at the center not to distract the user. The user can type and at the same time track what is written so far. At the bottom of the screen the sentence written so far by the user is presented, and at the top of the screen, the target sentence to be written by the user is viewed. The bottom cluster consists of 2 hexagons.

The first hexagon is '<', which represents the backspace, deletes the last character from the text box at the center. The second one is '>' represents the space or it also can be used to confirm the written word to be appended to the sentence written (previously confirmed words). Within each cluster, the first and last character of the cluster appears closest to the center of the screen. By looking at these two closest hexagons, the user can determine the range of characters covered by the cluster. The interactive clusters are arranged in a circular layout to help to reach the best differentiation

\footnotetext{
${ }^{1}$ The calibration was done by a user who did not take part in the experiment
}

between each of the interactive objects, as shown in figure 1a. The cluster and the hexagon (character) selection are done throughout a calibration-free selection.

\subsection{Display Dynamics}

The user can interact with the system's six interactive clusters through two interaction stages (two selections) [4]. The first interaction stage is to select a cluster and the second one is to select the desired character from the six characters which belong to the selected cluster or a word suggestion. These two interaction stages are done through three phases of the layout as shown in figure 1.

The objects' movement consists of five animations. Before the user detection gaze, the layout is stationary (phase 0). If a valid pursuit movement is detected, the clusters start rotation (first animation) to select a cluster (as shown in figures $1 \mathrm{a} \& 1 \mathrm{~b}$, then if a cluster is selected, the rotation of the clusters will stop. The selected cluster move outwards (second animation), phase 2, as shown in figure 1c. Phase 3 starts when the hexagons of the selected cluster start to move in distinct directions (third animation) from their directions within a cluster and also arranged in a circular layout as in figure $1 \mathrm{~d}$. This minimizes the variability in difficulty to follow objects' trajectories as all of the orientation of the objects is changed due to the animation. Also, these animations are like leading the user's eyes to know where to interact next. When the hexagons reach their new positions, they start rotation (fourth animation) to select a hexagon/character. In addition, another layer is added with the word suggestions which moves anti-clockwise "reverse animation than the hexagons "phase 4" (fifth animation), figure 1e". Then after the end of phase 4, phase 0 is initiated again in which the layout returns to its initial state and the word/character is written as seen in figure $1 \mathrm{f}$.

\section{EVALUATION AND RESULTS}

The experiment consists of one session. The participants start the experiment with one word training "Hello" and then the experimental task was to type 5 given phrases, 4 of the 5 phrases were proposed by MacKenzie and Soukoreff [10 ${ }^{2}$ and the fifth phrase was a popular English pangram ${ }^{3}$. The order of the phrases was randomized for each participant. Before the text entry user-interface is loaded, the phrases are displayed to the user in a message box. Then the session starts when the participant hits the start button of the shown message box. In addition, the required phrase is shows at the top of the screen so the participants can take a look at. The participants were told that if they made typos while writing, they are free to modify it or to leave it as it is. For the language model, we used a lexicon dictionary with 10.000 sentences [1] for the training Presage, the word prediction library [16]. At the end, the word with the highest probability is then added to the interface.

\subsection{Apparatus and Participants}

An HP laptop core i7 with $2.60 \mathrm{GHz}$ CPU and 16GB RAM ${ }^{4}$ used in the experiment with a screen resolution of $1024 \times 768$ pixels " 17

\footnotetext{
${ }^{2}$ My mother makes good cookies ., The force is with you !, Yes , this is a very good idea ., Did you have a good time?

${ }^{3}$ a sentence containing every letter of a given alphabet at least once "The quick brown fox jumps over the lazy dog."

${ }^{4}$ https://store.hp.com/us/en/mdp/laptops/envy-15-204072--1
} 


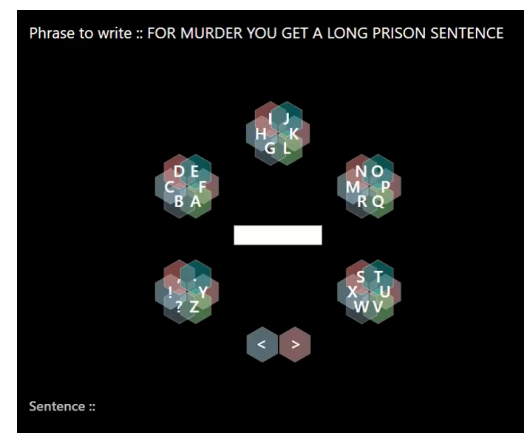

(a) Phase 0: Layout - Initial stationary layout, user wants to type ' $F$ '

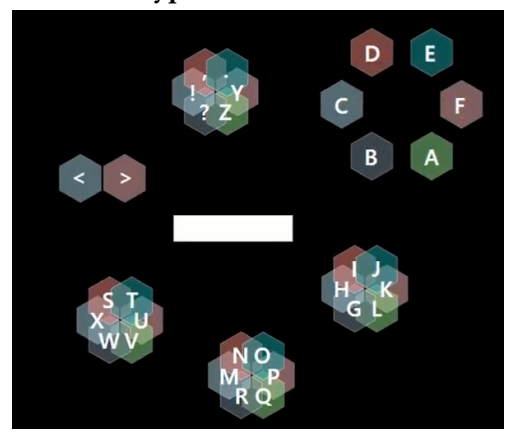

(d) Layout - The selected cluster's hexagons are animated and the word suggestions rotates to the other direction

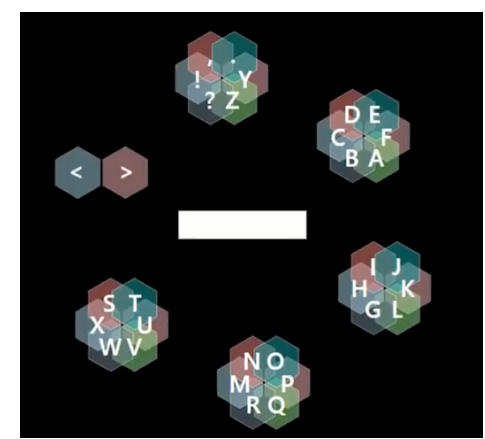

(b) Phase 1: Layout - User gaze is detected, rotation begun

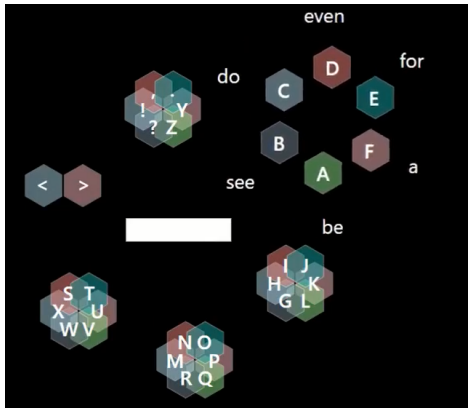

(e) Layout - Hexagons begun rotation, 'for' is selected \& typed in text box at the center

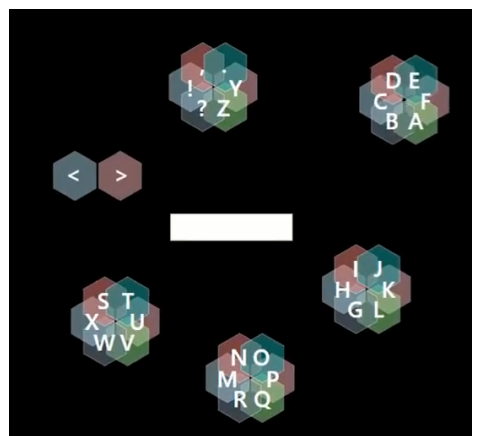

(c) Layout - The cluster which holds the ' $F$ ' is selected

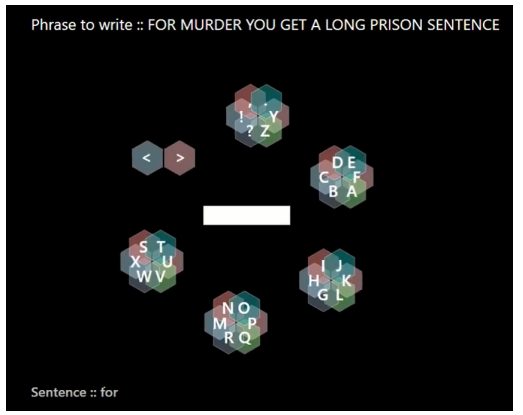

(f) Layout - After the 'for' is selected, selected cluster position is reset-ed to its position in the circular layout

Figure 1: System Phases

in". The laptop is running Windows 10. In addition, a Tobii 4C Eye Tracker ${ }^{5}$ is used to track the user's gaze on the display screen. The implementations was done using C\#. All of the participants were sitting steady and the distance between their eyes and the laptop screen was approximately about $40 \mathrm{~cm}$.

We invited 26 participants ( 10 male, 16 female) to contribute to the experiment. Participants aged between 16 and $29(\mathrm{M}=21.6$; $\mathrm{SD}=$ 2.5 ). Only three participants had experience with gaze-interaction. From the participant, only 8 had corrected vision using glasses. Participants were recruited by a word of mouth and had different backgrounds (middle school, high school, pharmacy, engineering, and computer science).

\subsection{Experiment Procedure}

Before beginning the experiment, each of the participants was told to sit steady and to keep their distance between their eyes and the laptop screen unchanged as possible as they can. Additionally, they were told that they can move their heads normally. Then the experiment aim was explained and the participants filled the consent and demographics forms. At the beginning of the experiment, the experimenter explained how the objects of the user interface act, move and illustrated the dynamics of the display in details. Also, the detailed steps to select a character or to select one of the suggested words are told to each of the participants. Then the participants

${ }^{5}$ https://help.tobii.com/hc/en-us/articles/213414285-Specifications-for-theTobii-Eye-Tracker-4C started their one-word trial where we didn't record the gaze data. After the experiment, a semi-structured interview took place in order to get qualitative feedback.

\subsection{Metrics Used for Evaluation}

In order to evaluate the system's usability and accuracy, we used a set of performance metrics. We also took into consideration the errors left in the sentences and the corrected errors made as explained in the following points:

- Typing Speed: measured in words per minute (WPM), where a word is any sequence of five characters, including letters, spaces, punctuation, etc.

- Minimum String Distance "MSD": the minimum distance between two strings defined in terms of editing primitives. The primitives are insertion, deletion, and substitution.

- Error Rate: calculated by comparing the transcribed text (text written by user) with the presented text, using the Minimum String Distance (MSD) method described by Soukoreff and MacKenzie [10]. This method does not take into account corrected errors.

- Keystrokes Per Character "KSPC": a measure of the average number of keystrokes used to enter each character of text. Ideally, $\mathrm{KSPC}=1.00$, indicating that each key press produces a character. If participants correct mistakes during entry, the KSPC value is greater than 1. For example, if "hello" is entered as h e l x (delete) $1 \mathrm{o}$, the final result is correct ( $0 \%$ error rate), but the KSPC value 
is $7 / 5=1.4$ ( 7 keystrokes to enter 5 characters). $\mathrm{KSPC}$ is an accuracy measurement reflecting the overhead incurred in correcting mistakes as described by Majaranta [11].

\subsection{Pilot Test}

A pilot test took place on 9 participants ( 4 male, 5 female) to test the system speed and the radius of moving hexagon's path. The participants aged between 18 and 25 (Mean $=21, \mathrm{SD}=2$ ). Only two participants had an experience with the eye-controlled interfaces. All participants had normal or corrected-to-normal vision.

The pilot test took place on 12 sentences randomly ordered for each participant from Soukoreff and MacKenzie's proposed dataset [10]. Each sentence was tested with different radius and speed. As a result, we choose 155 "pixels from the center" to be the radius

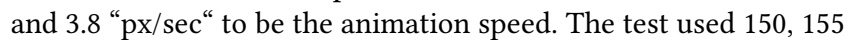
and 160 as radius and matched it with 4 different speeds 3.6, 3.8, 4 , 4.2. The bigger radius made it harder for the eyes to select the letters and the smaller ones were not accurate for the correlation to differentiate between them. For the speeds, the faster ones were harder for the eyes to follow and had a high error rate and the slower ones had lower typing speed and no error rate. For the small hexagons, we chose 65 as a radius.

\subsection{Results and Discussion}

The average typing speed of the system achieved is 3.41 WPM, the minimum typing speed achieved is $2.93 \mathrm{WPM}$ and the maximum typing speed is 3.86 WPM without training. Also, a typing of 4.7 WPM is reached as seen in figure 2. The average MSD error rate, which is the error rate of the uncorrected characters, is $3.04 \%$ "figure 3", and the average KSPC that calculates the error rates of the corrected words is 1.04 "figure 4". The error rates of the system are very low which means that the system is reliable and accurate in selecting characters, that most of the participants achieved KSPC = 1 and MSD error rate nearly $0 \%$ which is ideal. For the interviews, showed positive feedback from all participants and they assured that their usage to the system. They also reported that the system was easy to use and they learned to use it quickly. They also mentioned that by frequent usage they will be faster in typing.

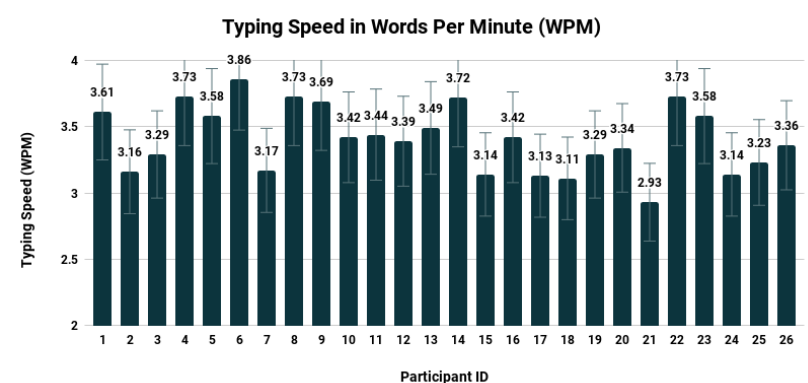

Figure 2: Overall Words Per Minute achieved per participant

3.5.1 Reflecting on the Literature. After described in the system with the results, now it is time to reflect on the literature found. It is clear that the main idea of this systems is taken from SMOOVS as mentioned previously, and hence, the similarities between both

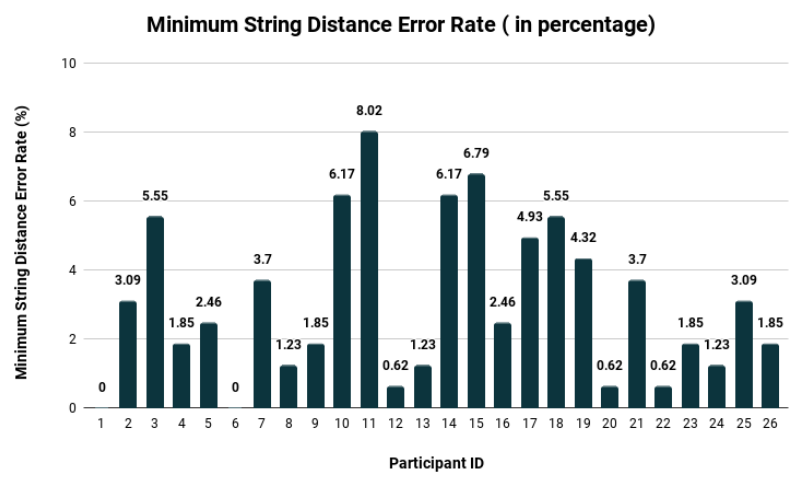

Figure 3: Minimum String Distance per participant

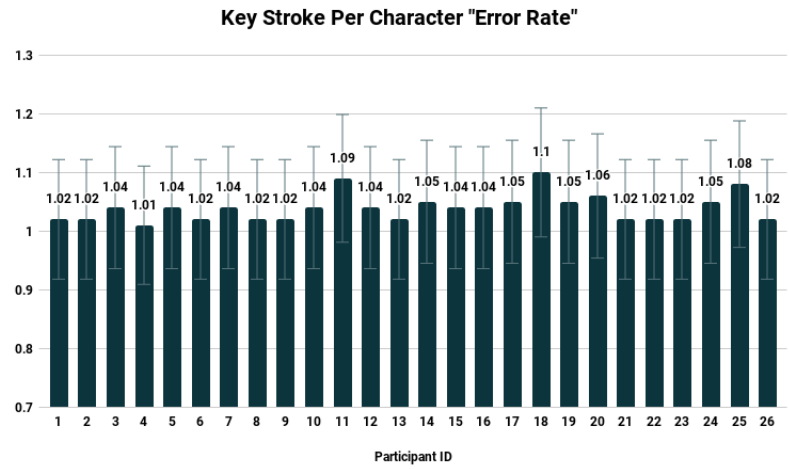

Figure 4: Keystroke Per Character for each participant

systems can be summarized into the following points. First, we accommodated the same layout and hexagon distributions. Second, we replicated the same movements for the big cluster along with the animations on how the hexagons separate from each other. On the other side, we added another level for word predictions along with cluster movements for the characters. In addition to the reverse movement for the prediction cluster. These are points regarding the layout. For the implementation, we are proposing a calibration free interaction with the system which will make it more usable on public displays and consumes shorter time for interaction with more WPM.

\section{CONCLUSION AND FUTURE WORK}

In this paper, we introduced a calibration-free gaze-based text entry based on smooth pursuits eye movements. Users were able to write with a 3.41 WPM as an enhancement over existing systems. We explained the system implementation and evaluation methodology along with the study. Qualitative feedback showed that the participants are willing to use it and the system perceived good usability. Finally, the system can be used when tactile or voice inputs are not feasible and it can also be in a good benefit to disabled users. In the future, we will enhance the motion correlation technique to reduce the error rate and reevaluate teh system with a bigger number of participants to calculate the learning curve. 


\section{REFERENCES}

[1] Featured on April 2018. Corpus of Contemporary American English.

[2] Nikolaus Bee and Elisabeth André. 2008. Writing with your eye: A dwell time free writing system adapted to the nature of human eye gaze. In International Tutorial and Research Workshop on Perception and Interactive Technologies for Speech-Based Systems. Springer, 111-122.

[3] George S Benson. 2011. Popular and influential management books. Useful research: Advancing theory and practice (2011), 289-308.

[4] Benjamin Blankertz, Matthias Krauledat, Guido Dornhege, John Williamson, Roderick Murray-Smith, and Klaus-Robert Müller. 2007. A note on brain actuated spelling with the Berlin brain-computer interface. In International Conference on Universal Access in Human-Computer Interaction. Springer, 759-768.

[5] Mohamed Khamis, Florian Alt, and Andreas Bulling. 2015. A field study on spontaneous gaze-based interaction with a public display using pursuits. In $A d-$ junct Proceedings of the 2015 ACM International foint Conference on Pervasive and Ubiquitous Computing and Proceedings of the 2015 ACM International Symposium on Wearable Computers. ACM, 863-872.

[6] Mohamed Khamis, Ozan Saltuk, Alina Hang, Katharina Stolz, Andreas Bulling, and Florian Alt. 2016. TextPursuits: using text for pursuits-based interaction and calibration on public displays. In Proceedings of the 2016 ACM International foint Conference on Pervasive and Ubiquitous Computing. ACM, 274-285.

[7] Andrew Kurauchi, Wenxin Feng, Ajjen Joshi, Carlos Morimoto, and Margrit Betke. 2016. EyeSwipe: Dwell-free text entry using gaze paths. In Proceedings of the 2016 CHI Conference on Human Factors in Computing Systems. ACM, 1952-1956.

[8] Michael F Land and Sophie Furneaux. 1997. The knowledge base of the oculomotor system. Philosophical Transactions of the Royal Society of London B: Biological Sciences 352, 1358 (1997), 1231-1239.

[9] Otto Hans-Martin Lutz, Antje Christine Venjakob, and Stefan Ruff. 2015. SMOOVS: Towards calibration-free text entry by gaze using smooth pursuit movements. Fournal of Eye Movement Research 8, 1 (2015).

[10] I. Scott MacKenzie and Shawn X. Zhang. 1999. The Design and Evaluation of a High-performance Soft Keyboard. In Proceedings of the SIGCHI Conference on Human Factors in Computing Systems (CHI '99). ACM, New York, NY, USA, 25-31. https://doi.org/10.1145/302979.302983

[11] Päivi Majaranta. 2009. Text entry by eye gaze. Tampere University Press.

[12] Päivi Majaranta, Ulla-Kaija Ahola, and Oleg Špakov. 2009. Fast gaze typing with an adjustable dwell time. In Proceedings of the SIGCHI Conference on Human Factors in Computing Systems. ACM, 357-360.

[13] Math.NET. [n. d.]. Math.NET. https://numerics.mathdotnet.com

[14] Carlos H Morimoto and Arnon Amir. 2010. Context switching for fast key selection in text entry applications. In Proceedings of the 2010 symposium on eye-tracking research \& applications. ACM, 271-274.

[15] Alex Poole and Linden J Ball. 2006. Eye tracking in HCI and usability research. Encyclopedia of human computer interaction 1 (2006), 211-219.

[16] Presage. [n. d.]. Presage. https://presage.sourceforge.io

[17] Stefan Schwarzkopf. 2015. Measurement devices and the psychophysiology of consumer behaviour: A posthuman genealogy of neuromarketing. BioSocieties 10, 4 (2015), 465-482.

[18] Korok Sengupta, Raphael Menges, Chandan Kumar, and Steffen Staab. 2017. Gazethekey: Interactive keys to integrate word predictions for gaze-based text entry. In Proceedings of the 22nd International Conference on Intelligent User Interfaces Companion. ACM, 121-124.

[19] Outi Tuisku, Päivi Majaranta, Poika Isokoski, and Kari-Jouko Räihä. 2008. Now Dasher! Dash away!: longitudinal study of fast text entry by Eye Gaze. In Proceedings of the 2008 symposium on Eye tracking research \& applications. ACM, 19-26.

[20] Antonio Diaz Tula, Filipe de Campos, and Carlos H Morimoto. 2012. Dynamic context switching for gaze based interaction. In Proceedings of the Symposium on Eye Tracking Research and Applications. ACM, 353-356.

[21] Mélodie Vidal, Andreas Bulling, and Hans Gellersen. 2013. Pursuits: spontaneous interaction with displays based on smooth pursuit eye movement and moving targets. In Proceedings of the 2013 ACM international joint conference on Pervasive and ubiquitous computing. ACM, 439-448.

[22] Michel Wedel and Rik Pieters. 2008. A review of eye-tracking research in marketing. In Review of marketing research. Emerald Group Publishing Limited, 123-147. 